Article publié dans L’Enseignement philosophique, 69-4, juin-juillet 2019, pp. 33-51 (les nombres entre crochets correspondent à la pagination dans la revue).

\title{
Quelques malentendus au sujet de l'antispécisme
}

\section{Analyse de l'argumentation de Philippe Larralde dans son article concernant l'antispécisme}

\author{
Malo Morvan \\ Université de Rennes 2 \& CERLIS, Paris Descartes \\ « En effet il critique une telle idée [...] en méconnaissant \\ gravement la philosophie qu'il a l'illusion de dépasser. » \\ Philippe Larralde, 2018
}

Je souhaiterais ici revenir sur l'article de Philippe Larralde intitulé «La critique du "spécisme" comme illusion métaphysique : pour un regard critique sur notre relation aux animaux non humains. ", paru dans le numéro précédent de L'enseignement philosophique (69-3).

Indépendamment des thèses défendues par l'auteur, je souhaiterais ici revenir sur quelques considérations de méthode. Autrement dit, mes remarques critiques visent moins le fait qu'il s'oppose à l'antispécisme que les arguments qu'il mobilise à cette fin, qui me semblent manquer de rigueur.

Le point de départ de cette relecture est un agacement à voir un de mes articles ${ }^{1}$ réutilisé d'une manière qui m'a semblé manifester peu d'honnêteté intellectuelle. Celui-ci a fait usage de certaines citations que je donne de textes antispécistes : celles-ci sont d'ailleurs les seules citations de littérature antispéciste que l'auteur donne dans tout son article ${ }^{2}$ : à mon sens cela en dit long sur son degré d'information au sujet du courant qu'il critique ; par ailleurs, leur usage est en partie détourné de leur sens initial. Mon [34] agacement réside donc dans le fait que mon article ait pu servir de caution à une démarche intellectuelle que je ne cautionne pas, et ce pour des raisons qui relèvent moins du contenu du débat en lui même (faut-il ou non se déclarer "antispéciste" ?) que de la déontologie dans l'acte d'écriture. Malgré cet agacement, je vais tenter, autant que faire se peut, de ne pas m'engager dans une escalade par trop polémique qui nuirait à la qualité de l'argumentation : je me contenterai donc généralement de mentionner les écarts entre ce que l'auteur fait dire à l'antispécisme (ainsi qu'à l'utilitarisme) et ce que ce courant dit réellement (en prenant en compte, dans leurs grandes lignes uniquement, les divergences au sein de ce courant), et je laisserai au lectorat le soin de juger si un tel écart est dû à une absence d'information, ou à une volonté délibérée de déformer un propos (ainsi que toute question subsidiaire, comme l'éventualité que ces deux dimensions coexistent, ou celle de savoir laquelle serait la plus fâcheuse).

1 M. Morvan 2016, , «Ensemble dans la sentience, seuls dans la sentence. Analyse des processus de différenciation/identification entre espèces humaine et non-humaines dans le discours antispéciste », in Tétralogiques, $\mathrm{N}^{\circ} 21$, Existe-t-il un seuil de l’humain ?, pp. 247-286. http://www.tetralogiques.fr/spip.php?article33

2 La citation qu'il donne de Paola Cavalieri se trouve p. 264 de mon article, et les deux citations qu'il donne de Singer proviennent de la p. 253 de celui-ci. L'auteur interprète la citation de Cavalieri comme relevant d'une « erreur grossière » essentiellement car il lui attribue une position visant à nier une particularité humaine, alors que l'expression précise d' « égale considération des intérêts » chez Cavalieri montre bien que la question ne porte pas ici sur l'identification / différenciation entre les espèces (cf. la première section du présent article). De même, les deux citations de Singer prises dans mon article auraient dû mener Philippe Larralde à revenir sur les formulations réductrices de sa section III selon lesquelles Singer défendrait que la vie d’un animal non-humain vaudrait plus que celle d'un humain : l'auteur mobilise ainsi des citations qui entrent en contradiction avec le propos qu'il tenait luimême au sujet de Singer quelques pages plus tôt. 


\section{Mauvaise identification de la thèse antispéciste}

Le premier et principal défaut de l'article de Philippe Larralde est qu'il ne donne pas une définition correcte de la posture à laquelle il s'oppose. En particulier, dans sa critique de l'antispécisme, il confond deux dimensions : d'une part, la question, que l'on pourrait nommer logique (au sens où elle porte sur une opération d'identification ou de différenciation entre deux ensembles) métaphysique, ou encore anthropologique, de la différence entre espèces humaine et non-humaines, d'autre part, la question, éthique ou politique, de la revendication d'une équité dans la considération de leurs intérêts. Or, il est possible de revendiquer la seconde dimension, politique, sans pour autant nier, d'un point de vue anthropologique, ou métaphysique, une particularité ou une spécificité de l'espèce humaine.

Il faut donc être très clair sur un point de définition : l'antispécisme n'est pas une position qui nierait l'existence d'une particularité de l'espèce humaine par rapport à d'autres espèces (question logique-métaphysique-anthropologique), mais seulement une position qui revendique, sous certaines conditions, une équité de considération dans l'intérêt des êtres vivants à ne pas souffrir, indépendamment de l'espèce à laquelle ils appartiennent (question éthique-politique). À la limite, on pourrait nommer "différentialiste" et "anti-différentialiste" l'alternative renvoyant à la première question, le premier courant défendrait une différence essentielle de l'espèce humaine, le second une continuité envers les autres espèces. On pourrait aussi observer qu'une question posée en des termes aussi massifs risque fort de mener à des apories, et qu'il peut exister une diversité de manières de catégoriser le jeu logique des identifications / différenciations interespèces ${ }^{3}$. L'essentiel pour notre propos est simplement le point suivant : il est possible d'être à la fois différentialiste (défendre une particularité de l'humain) et antispéciste, il n'y a pas d'incompatibilité logique entre ces deux positions, car elles ne répondent pas aux mêmes questions. Une telle posture, majoritaire dans le mouvement antispéciste, se formulerait ainsi : bien que l'espèce humaine soit logiquementmétaphysiquement-anthropologiquement différente des autres espèces, il est néanmoins légitime de réclamer, éthiquement-politiquement, de prendre en compte avec équité la capacité de certaines autres espèces à ressentir de la douleur, lorsque cela est pertinent.

Faute d'être au clair sur cette définition, on ne peut que s'embourber dans des confusions. L'article de l'auteur en fourmille, voici quelques exemples où il présente la thèse antispéciste : [35]

- « l'idée selon laquelle il n’y aurait pas lieu d'affirmer une spécificité de l'homme ${ }^{4}$

- « la négation, au nom de notre proximité avec les animaux, de la spécificité de l'homme, »

- « [Singer] nomme l'affirmation d'une spécificité humaine (qui est bien sûr une supériorité impliquant un absolu respect) le "spécisme" »

- «La négation de cette spécificité constitue ainsi une profonde régression intellectuelle. »

À ce sujet, je voudrais ajouter deux remarques. Premièrement, ce n'est en réalité pas tellement la question de la différence entre l'espèce humaine et les autres espèces qui est en jeu. En effet, puisque toutes les espèces diffèrent les unes des autres, la question n'est pas de savoir si l'espèce humaine diffère des autres, mais bien si la manière dont nous différons des autres espèces diffère de celle dont les autres espèces diffèrent entre elles (il s'agit donc d'une différence au carré, ou différence portant sur la différence). Par exemple, les pieuvres possèdent trois cœurs, les kangourous ont une poche abdominale, et les chauves-souris s'orientent avec des ultra-sons : voilà

3 On s'intéressera notamment à l'ouvrage de Philippe Descola, Par-delà nature et culture, Gallimard, 2005, devenu un classique. L’auteur y distingue schématiquement différentes manières de penser les rapports entre espèces humaine et non humaine, selon les diverses sociétés : animisme, totémisme, naturalisme, analogisme.

4 Toutes les citations présentées de cette manière, sans référence, sont extraites de l'article de Philippe Larralde. Les italiques sont de l'auteur, les caractères gras sont de mon fait lorsque je souhaite souligner une formulation précise. 
des particularités propres à chaque espèce (ou à peu près). Néanmoins, tout ce que l'on peut dire à partir de tels constats, c'est que les espèces diffèrent les unes des autres. Ce qu'il faudrait parvenir à expliquer, ce serait que l'une de ces particularités ait un statut différent, plus important, que celles qui existent déjà d'une espèce à une autre et les différencient entre elles. Autrement l'on se voit condamnés à ravaler sur le même rang des constats de type "les humains sont la seule espèce capables de X" et "les chauves-souris sont la seule espèce capable de s'orienter par ultra-sons". Ainsi, lorsque l'on met en avant telle ou telle particularité de l'humain (le langage, le rire, la raison, l'érotisme, la liberté, la conscience, etc.), il reste encore à expliquer en quoi cette singularité représenterait plus qu'une simple différence d'une espèce animale à une autre.

Deuxièmement, non seulement la question logique-métaphysique-anthropologique de la différence (ou différence de différence) entre les espèces peut être distinguée de celle, éthique-politique, de l'équité de traitement entre les espèces, mais on peut même soutenir qu'il est nécessaire de soutenir une particularité éthique de l'espèce humaine pour revendiquer politiquement qu'elle prenne en considération les intérêts des autres animaux, alors que les mammifères carnivores, par exemple, ne sont pas soumis à une telle injonction : les antispécistes considèrent comme immoral que des humains, qui seraient capables de faire autrement, et qui ont conscience de leurs actes, tuent et fassent souffrir volontairement d'autres animaux pour leur alimentation, mais pas qu'un animal carnivore en mange un autre quand cela relève de son instinct et de son régime alimentaire ${ }^{5}$. C'est un tel argument que je défends dans mon article, à partir d'une analyse des procédés discursifs de différenciation et d'identification présents au sein d'un corpus de textes antispécistes. Philippe Larralde a également eu une intuition de cette idée selon laquelle il était nécessaire de supposer chez les humains une capacité éthique spécifique, pour leur demander un comportement que l'on ne demanderait pas à d'autres espèces (se priver de manger de la viande), mais dans la mesure où il s'obstine à confondre entre elles les questions logique-métaphysique-anthropologique de l'identité / différence et celle, éthique-politique, de l'équité de traitement, il en déduit une " autocontradiction pragmatico-transcendantale » au sein du discours antispéciste : [36]

« [Demander aux humains de ne plus manger de viande,] Cela revient bien sûr à affirmer hautement la spécificité (supériorité) de l'homme qu'est la liberté (alors que l'on prétend explicitement le contraire), et à considérer qu'il y a une dignité de l'homme unique dans le règne animal, laquelle légitime le respect absolu qui n'est dû qu'à lui. En un mot, c'est le comble du "spécisme" ! »"

La remarque selon laquelle une telle démarche constitue " le comble du "spécisme" » se veut peutêtre un trait d'humour, mais elle marque surtout une profonde incompréhension des notions de "spécisme" et d'"antispécisme". Dans la mesure où la définition de ces notions est relativement facile à trouver dans n'importe quel ouvrage d'introduction consacré à ce sujet, elle marque en définitive une véritable absence d'information quant au domaine étudié. À cet égard, Philippe Larralde semble mener une politique du "plus c'est gros, plus cela passe", dans certaines de ses formules franchement caricaturales :

«Assez étrangement les défenseurs antihumanistes (utilitaristes) des animaux semblent généralement très disposés à admettre que seul l'homme est capable de choisir le mal comme projet, d'accomplir le mal pour le mal. Ils y voient une raison de rabaisser son statut (il se croit supérieur alors qu'il se montre au fond inférieur aux autres animaux); $»$

5 Il arrive néanmoins que les débats autour de l'antispécisme évoquent la question de savoir si nous devrions ou non intervenir pour réguler la prédation naturelle (cf. Steve Sapontzis ou André Méry), mais les positions favorables à une telle intervention sont assez marginales, et elles s'appuient encore sur notre capacité spécifique d'humains à intervenir dans les écosystèmes.

6 On trouve une autre formulation analogue, témoignant de la même confusion, ailleurs dans le texte (je souligne) : " De même, lorsque les utilitaristes (se rendant au passage coupables de "spécisme ») expliquent que c'est justement parce que l'homme est supérieur aux animaux qu'il doit se comporter moralement envers eux, c'est exactement ce que dit Kant. Nul besoin pour cela de nier la distinction essentielle homme (liberté)-animal (nature). ". 


\section{Association entre capacité et respectabilité}

Nous avons vu que la distinction entre la question question logique-métaphysique-anthropologique du différentialisme et celle, éthique-politique, du spécisme, avait pour conséquence qu'il est possible de défendre une prise en compte des intérêts qu'ont les autres espèces animales à vivre décemment sans pour autant nier la particularité des humains. C'est une distinction que Philippe Larralde ne semble pas concevoir (ou seulement sous la forme d'une « autocontradiction pragmatico-transcendantale ») ; il insiste ainsi fortement sur l'affirmation d'une particularité de l'humain, comme si cela avait une quelconque chose à voir avec la question du spécisme. Mais surtout, il répète régulièrement que cette spécificité des capacités humaines constitue le fondement de l'exigence morale envers les humains ; voici un relevé non exhaustif de formules répétitives allant en ce sens (je souligne) :

- «la négation, au nom de notre proximité avec les animaux, de la spécificité de l’homme, laquelle fonde le respect absolu qui n'est dû qu'à lui »

- « la claire conscience d'une proximité ne doit pas nous interdire de penser la spécificité de l'homme et le respect absolu qui lui est dû, lequel découle de cette spécificité »

- «Il apparaît ainsi que le fondement d'un point de vue critique sur notre relation aux animaux non humains est la pensée claire (et non dogmatique) de la spécificité de l'homme en tant qu'il relève seul du droit. »

- « si l'utilitarisme est le principe de la régression antihumaniste, c'est parce qu'il refuse l'idée d'une spécificité de l'homme, laquelle fonde un respect absolu qui n'est dû qu'à lui. »

- «On le voit, le danger mortel, d'un point de vue moral, réside dans le renoncement au respect absolu qui, d'un point de vue humaniste, est dû à toute vie humaine. Telle est la terrible conséquence du refus utilitariste d'admettre une spécificité de l'homme en fonction de laquelle il est le seul être vivant qui doit toujours être considéré comme une fin en soi, ce qui fonde le respect absolu qui n'est dû qu'à lui. »

Une telle association répétée entre ces deux dimensions révèle un présupposé : celui selon lequel la respectabilité morale d'un être vivant dépendrait de certaines de ses capacités, en l'occurrence des capacités propres aux humains, que l'on nommera "raison", [37] "liberté", ou autre, dont on affirmerait qu'elles ne sont pas partagées par les autres espèces. Autrement dit, c'est parce qu'un être vivant serait plus intelligent qu'il serait justifié d'exiger de ne pas le faire souffrir. Selon cette perspective, si un être vivant était encore capable de souffrir, mais néanmoins dénué de l'intelligence attribuée aux humains, alors il serait acceptable moralement de le faire souffrir. Dans un tel discours, le critère qui fonde l'exigence de respectabilité morale d'un être vivant est son intelligence (ou quelqu'autre caractère que l'on ait pu mettre en avant pour justifier une "spécificité" : liberté, rire, érotisme, etc.), plus que sa capacité à ressentir peine et douleur.

Si les thèses de l'antispécisme s'opposent à celles de Philippe Larralde, c'est, bien plus que sur la question d'une spécificité humaine, sur ce présupposé associant respectabilité morale et capacités intellectuelles. Peter Singer formule ainsi sa position :

« [...] si nous devions choisir de faire cesser la douleur soit chez un humain normal soit chez un humain handicapé mental - si par exemple tous deux souffrent de blessures douloureuses mais superficielles, sans que nous ne disposions d'assez d'analgésiques pour les deux - il est loin d'être aussi simple d'opérer un choix. Cela reste vrai si nous considérons d'autres espèces. $\rangle^{7}$

S'il y a bien un point qui caractérise la particularité du discours utilitariste, c'est de remarquer que le critère qui fonderait l'exigence de ne pas infliger de la douleur à un être vivant devrait résider davantage dans la capacité de cet être à ressentir ou non cette même douleur, que dans celle de

7 P. Singer, 1975, La libération animale, trad. Payot 2012, pp. 94-95 
savoir quelle est la forme d'intelligence dont il dispose. Comme le dit Bentham : « La question n'est pas : Peuvent-ils raisonner ? Ni : Peuvent-ils parler ? Mais : Peuvent-ils souffrir ? $»^{8}$.

Alors que Philippe Larralde cite Rousseau parmi les auteurs au nom desquels on peut revendiquer un "humanisme"', on relira utilement cet extrait du Discours sur l'origine et les fondements de l'inégalité parmi les hommes :

« L'homme est assujetti envers [les autres animaux] à quelque espèce de devoir. Il semble, en effet, que si je suis obligé de ne faire aucun mal à mon semblable, c'est moins parce qu'il est un être raisonnable que parce qu'il est un être sensible ; qualité qui, étant commune à la bête et à l'homme, doit au moins donner à l'une le droit de n'être point maltraitée inutilement par l'autre. »

Si Philippe Larralde s'était donné les moyens d'aborder avec rigueur les discours antispéciste et utilitariste (dont nous verrons plus loin qu'ils ne sont pas nécessairement associés), il aurait perçu que le nœud du désaccord réside moins dans la négation d'une particularité humaine que dans le déplacement $d u$ critère d'exigence morale d'une capacité intellectuelle vers une capacité à ressentir de la douleur ${ }^{10}$. [38]

Quoi qu'il en soit, il demeure cohérent avec une telle association lorsqu'il déclare qu'il est acceptable d'élever et de tuer des porcs et des poulets pour les manger, mais pas des chimpanzés et des gorilles, dont l'intelligence les rapproche des humains ${ }^{11}$ :

« La conséquence pratique de cela est sans doute que ceux-ci méritent d'être traités avec un respect tout particulier, notamment les grands singes. Bien entendu nous avons un devoir moral de ne pas faire souffrir inutilement les animaux en général, mais, par exemple, alors que nous mangeons du porc ou du poulet, il semble inacceptable, sur le plan éthique, de manger un chimpanzé ou un gorille, précisément en raison de leur proximité avec l'homme. $»^{12}$

8 J. Bentham, An introduction to the Principles of Morals and Legislation, ch. 17. L'antispécisme contemporain s'est doté de la notion de "sentience" pour rendre compte de cette capacité d'un être vivant à ressentir les sensations physiologiques de peine et de douleur en se débarrassant d'une dimension spiritualiste dans la description de ces états, qui permettait encore naguère de tenir des propos spécieux de type : "certes, un chien que l'on frappe peut avoir mal, mais il n’a pas conscience qu'il a mal.". Cf. en particulier les travaux de Gary Francione à ce sujet.

9 On remarquera au passage qu'il ne prend jamais le soin de définir la notion d'"humanisme", et donc de dissiper la grande polysémie qu'il abrite, l'"humanisme" d'un Pic de la Mirandole ou d'un Emmanuel Mounier ayant pourtant peu de choses à voir avec celui d'un Sartre.

10 Comme nous le verrons, l'argument singerien des cas-limites chez les humains sert précisément à court-circuiter cette association opérée entre capacité et respectabilité : si vous considérez que c'est l'intelligence qui justifie l'exigence de ne pas faire souffrir, alors que faites-vous des humains qui ne disposent pas d'une telle intelligence ? Si vous souhaitez maintenir l'association entre capacité et respectabilité, alors il vous faut accepter que l'on puisse les faire souffrir autant que les mammifère d'élevage, mais si (très vraisemblablement) vous refusez une telle conséquence, c'est qu'implicitement vous avez fondé votre exigence morale envers ces humains sur leur seule capacité à souffrir, et, en toute logique, un tel déplacement de ce critère vous obligera à prendre en compte cette même capacité à souffrir chez les mammifères d'élevage.

11 Le numéro 8 des Cahiers antispécistes (http://www.cahiers-antispecistes.org/numero/08/) propose plusieurs articles pour discuter ce point, à partir du "Projet Grands Singes". La ligne générale à ce sujet pourrait se résumer ainsi : puisque c'est bien la sentience qui fonde l'exigence de ne pas infliger de douleur, et non l'intelligence, alors il n'y a pas de raison d'accepter de traiter différemment des êtres sentients plus intelligents (certaines espèces de singes) et les autres. Tout au plus, la répugnance inspirée par le fait de voir souffrir des êtres envers lesquels une identification serait plus facile devrait nous inciter à une généralisation envers les autres espèces sentientes moins ressemblantes.

12 On notera un usage intéressant du partitif pour désigner les animaux qu'il estime légitime d'élever pour les tuer : « $\underline{\mathrm{du}}$ porc, $\underline{\mathrm{du}}$ poulet » : les être vivants sont ainsi décrits comme des quantités indifférenciées, renvoyant davantage à la viande que l'on en retire qu'aux êtres vivants que l'on élève. En revanche, les grands singes sont désignés par un article défini («manger un chimpanzé ou un gorille ») : où l'on voit ainsi une prise en compte de leur individualité. L'impression subjective sur le lectorat est sûrement différente si l'on écrit : "manger un porc ou un poulet" / "manger du gorille ou du chimpanzé". 


\section{Défense par l'invocation des "droits" et défense par l'utilitarisme :}

Une troisième erreur factuelle dans l'article de Philippe Larralde consiste à tenir pour équivalents une défense utilitariste des animaux non-humains et une défense revendiquant des "droits des animaux" :

- «Or, au-delà même de cette illusion selon laquelle il y aurait des "droits des animaux", le principe de la régression intellectuelle tendant à effacer la frontière homme-animal est le point de vue utilitariste que suppose cette illusion. »

- «Telle est l'erreur de raisonnement des utilitaristes : si vous affirmez que l'homme, comme seul être de liberté, est le seul à pouvoir être considéré comme personne juridique (les animaux n'ayant pas de droits), alors vous ne pouvez pas concevoir de prise en compte des intérêts des animaux non humains. »

Historiquement, on constate que l'éthique animale contemporaine s'est fondée sur un clivage important entre deux courants de pensée : le premier, défendu par Peter Singer dans Animal Liberation (1975), repose sur une base utilitariste (et pour être plus précis : un utilitarisme des préférences) ; le second, défendu par Tom Regan dans The Case for Animal Rights (1983), défend une position qu'il est convenu d'appeler "droit des animaux". Ainsi, l'approche par l'utilitarisme et l'approche par les droits sont bien deux courants concurrents au sein du mouvement antispéciste, et les confondre relève d'une grave erreur. On trouve ainsi un article de Peter Singer, paru en 1987, intitulé "Animal Liberation or animal rights? ${ }^{13}$, où l'auteur explique pour quelles raisons il ne souscrit pas à l'expression de "droit des animaux".

Au sujet de ces mêmes droits, une connaissance même sommaire des thèses antispécistes aurait permis à l'auteur de savoir qu'il s'agit moins de théoriser des "droits des [39] animaux" que d'interroger les "animaux dans le droit" : ainsi, le point de départ de Gary Francione est bien le constat que les animaux non-humains ont le statut de biens meubles dans le droit, ce qui ne rend pas compte de leur sensibilité d'être vivants; les réflexions de Martha Nussbaum visent à intégrer les animaux non-humains dans l'approche de l'éthique des capabilités qu'elle a élaborée avec Amartya Sen ; le récent ouvrage Zoopolis de Donaldson et Kymlicka vise à proposer des pistes constructives d'adaptations juridiques qui prendraient en compte les rapports que nous avons envers les différentes espèces (domestiques, sauvages, liminaires), en revendiquant explicitement s'intéresser moins à un "droit des animaux" qu'à nos responsabilités à leur égard. Ainsi, il n'est pas nécessaire d'attribuer des "droits" aux autres animaux pour envisager la possibilité que nous ayons des "devoirs" à leurs égards. Si Philippe Larralde mentionne bien la distinction classique entre "agent moral" (capable de contracter ou d'être responsable de ses actes, dont on peut exiger qu'il ait des devoirs) et "patient moral" (envers qui nous avons des devoirs), il semble ne pas en tirer les conséquences pour l'antispécisme, puisqu'il continue à déclarer que réfléchir à nos responsabilités envers d'autres espèces impliquerait forcément d'attribuer des droits à ces dernières. Or, à ma connaissance, aucun courant antispéciste ne revendique que les animaux non-humains devraient être considérés comme des agents moraux ${ }^{14}$.

13 P. Singer, 1987, « Animal Liberation or animal rights ? », The Monist, n70/3, pp. 3-14. Le texte est traduit dans Philosophie Animale, Vrin (collection "textes clés"), 2010, pp. 137-160. Il affirmait déjà dans La Libération Animale : «L'emploi du vocabulaire des droits représente un raccourci politique pratique [...] mais dans l'argumentation en faveur d'un changement radical dans notre attitude envers les animaux, ce langage n'est en rien nécessaire. » (op. cit., p. 76).

14 Comme déjà mentionné, il existe bien au sein du mouvement de réflexion antispéciste un courant qui s’interroge sur la prédation dans le monde sauvage, sous le nom de RWAS (Reducing Wild-Animal Suffering). Mais même alors, lorsque les auteurs parlent d'interventions humaines au sein du monde sauvage pour limiter la douleur qu'implique la prédation (cf. Steve Sapontzis (http://www.cahiers-antispecistes.org/faut-il-sauver-le-lievre-du-renard/) ou André Méry (http://www.cahiers-antispecistes.org/sur-la-predation-reponse-a-philippe-laporte/)), c'est au nom d'une responsabilité humaine, et de la prise en compte du pouvoir qu'aurait notre espèce d’intervenir, et donc des responsabilités que cela engendre. Cf. les nn. 40 et 41 des Cahiers Antispécistes (http://www.cahiersantispecistes.org/numero/40/, http://www.cahiers-antispecistes.org/numero/cahiers-antispecistes-n41-eliminer-les- 
C'est donc en toute logique qu'il défend la thèse selon laquelle, si l'on conférait des "droits" à un animal, on lui imputerait également des "devoirs" (il serait ainsi impossible de le considérer comme simple "patient moral" sans qu'il ne soit réciproquement "agent moral"), ce qui renverrait selon lui à la tradition médiévale absurde des procès d'animaux. Outre les animaux non-humains, les cas sont pourtant communs de séparation entre les deux qualités : les enfants en bas âge, personnes âgées séniles, les personnes mentalement déficientes, dans le coma, voire des entités plus abstraites comme "les générations futures" ou "l'écosystème" peuvent être considérés comme des patients moraux (au sens où nous nous attribuons des obligations morales à leur égard) sans pour autant être des agents moraux (nous savons ne pas pouvoir attendre d'eux qu'ils agissent moralement) ${ }^{15}$. Ainsi l'affirmation de l'auteur " on ne saurait concevoir de droits sans devoirs », soulignée par lui-même, se heurte-t-elle à de nombreux contre-exemples au quotidien, indépendamment de la question animale. La présentation des procès d'animaux non humains se situe dans la même logique : elle repose sur l'adéquation suivante : "si les animaux devaient avoir des droits, alors on pourrait aussi les juger", et on peut ici lui opposer la même liste d'humains que l'on considère comme difficilement condamnables devant la loi (enfants, personnes mentalement déficiente, générations futures, etc.), ce qui ne les empêche pas, heureusement, d'avoir des droits. Malgré l'ensemble d'associations approximatives sur lesquelles elle repose, la mention [40] des procès d'animaux n'en a pas moins un effet rhétorique certain, en proposant d'associer la revendication antispécistes à des pratiques moyenâgeuses aujourd'hui jugées risibles.

\section{Association de l'antispécisme à l'utilitarisme}

La quatrième erreur de Philippe Larralde, qui bizarrement n'est pas incompatible avec la troisième, réside dans l'association qu'il opère entre utilitarisme et antispécisme, comme si le premier était la seule manière possible de défendre le second (sauf lorsqu'il est confondu avec une théorie des droits des animaux). Si Peter Singer est à la fois un défenseur de la cause animale et un utilitariste, on pourrait dire que le lien entre les deux ne va pas beaucoup plus loin. En particulier, le champ de l'éthique animale est marqué par une grande diversité de propositions théoriques parmi lesquelles l'utilitarisme n'est qu'une option possible. Nous avons déjà mentionné Tom Regan, qui a historiquement constitué le second pilier important sur lequel s'est édifié l'antispécisme contemporain, et qui est loin d'être utilitariste. Mais il existe d'autres fondements possibles pour défendre l'antispécisme, et les autres auteurs que nous avons mentionnés (Gary Francione, Martha Nussbaum, Donaldson \& Kymlicka, etc.) fondent également leurs approches antispécistes sur d'autres bases que l'utilitarisme. En vérité, si l'on voulait rendre compte de la totalité des courants visant à défendre l'antispécisme, il faudrait prendre en compte divers jeux d'oppositions, notamment entre abolitionnistes (vegans et partisans de l'arrêt de l'élevage, de l'expérimentation animale, etc.) et welfaristes (qui défendent le bien-être animal et pour qui l'amélioration de conditions de vie pourrait suffire); entre les courants voulant fonder l'éthique animale sur une argumentation rationnelle et ceux revendiquant de s'appuyer davantage sur la dimension des émotions ou s'inspirant d'une éthique du care, etc. La lecture de n'importe quel ouvrage d'introduction à l'éthique animale aurait permis à l'auteur d'éviter une telle confusion entre antispécisme et utilitarisme ${ }^{16}$.

animaux-pour-leur-bien/) à ce sujet.

15 On pourrait encore élargir la liste des patients moraux si l'on prend en compte les discours invoquant des devoirs envers la Patrie, envers une Culture, une Langue, une Identité, etc. Les discours anti-avortement invoquant un "droit du fœtus" considèrent également celui-ci comme un patient moral sans encore pouvoir être un agent moral, etc..

16 Pour un aperçu de la diversité des positions, on pourra lire J.-B. Jeangène Vilmer, « Les principaux courants en éthique animale », in. La question animale, entre science, littérature et philosophie, PUR, 2011, pp. 79-92 (disponible aussi ici : http://www.jbjv.com/Les-principaux-courants-en-ethique.html). En plus des courants déjà mentionnés, l'auteur en évoque d’autres s’appuyant sur des positions religieuses, scientifiques, environnementales, ou féministes. On consultera également utilement l'ouvrage Philosophie Animale paru dans la collection "textes clés", Vrin, 2010, et particulièrement la deuxième partie sur l’éthique. 
Il pourrait sembler exagéré de dire qu'il existe autant de théories antispécistes qu'il existe de courants théoriques en éthique, mais cela ne serait sûrement pas très éloigné de la diversité actuelle des fondements théoriques à cette revendication. De même, nous pourrions dire qu'il existe entre l'antispécisme et l'utilitarisme le même rapport qu'entre l'éthique et la méta-éthique : alors que le premier dit quoi faire, le second en explicite les raisons théoriques. On peut donc se retrouver, d'un point de vue éthique, sur la question de savoir ce qu'il convient de faire ou non, tout en divergeant par ailleurs sur les raisons théoriques qui fonderaient de telles maximes pratiques. Bref, l'antispécisme n'est nullement contraint dans sa formulation par un cadre utilitariste (les termes "utilitariste" et "utilitarisme" font l'objet de 57 occurrences dans l'article de l'auteur, qui entend pourtant évoquer l'antispécisme en général).

En fait, l'utilitarisme détermine si peu la formulation de l'antispécisme qu'il est également possible de défendre des positions spécistes sur des bases utilitaristes : c'est le cas de Raymond Frey, qui est plus précisément utilitariste des préférences. Dans son ouvrage Interests and Rights: The Case Against Animals (1980), l'auteur s'oppose à Singer [41] en défendant que les "préférences" ou intérêts reposent sur des désirs, qui s'appuient sur des croyances qui ne peuvent elles-mêmes être élaborées que si l'on dispose du langage ; or les animaux non-humains ne disposant pas du langage, ils ne pourraient formuler de préférence ${ }^{17}$.

Si une telle association entre antispécisme et utilitarisme constitue une grave erreur lorsqu'elle est présentée comme nécessaire ou intrinsèque, elle n'en produit pas moins un effet rhétorique efficace dans l'économie argumentative de l'auteur, grâce à une succession de glissements qu'il opère par association: de l'antispécisme à l'utilitarisme, au conséquentialisme, à l'hétéronomie, pour en arriver à présenter l'antispécisme comme nécessairement une morale de l'hétéronomie. Un tel ensemble de glissements lui permet habilement d'associer son antagoniste à la figure de l'enfant, dans la mesure où, s'appuyant sur Piaget, il s'autorise une identification entre "conséquentialisme" et "morale enfantine"18.

\footnotetext{
« Le problème est au fond qu'une morale qui juge sur les conséquences est, par essence, enfantine, alors que seule une morale jugeant sur l'intention peut être une morale adulte. C'est ce qu'a parfaitement montré J. Piaget (Le jugement moral chez l'enfant). [...] Il apparaît donc que, par principe, la morale utilitariste, parce qu'elle est conséquentialiste, présente les caractéristiques d'une morale qui n'est pas encore adulte. »
}

Par le jeu des identifications, l'exigence morale des antispéciste se trouve ainsi assimilée au caprice d'enfant qu'il faudrait encore éduquer pour lui enseigner la véritable morale, celle des adultes. Il est probable que, dans le cadre des débats contemporains en éthique et méta-éthique, tou·te·s les auteur'e's qui, antispécistes ou non, utilitaristes ou non, souhaitent prendre en compte la dimension des conséquences pour évaluer des actes moraux, apprécieront de se voir ainsi littéralement infantilisé $\cdot e \cdot s$.

\section{Présentation caricaturale de l'utilitarisme}

La cinquième difficulté dans la démarche de l'auteur réside en ce qu'il ne se contente pas d'associer l'antispécisme à l'utilitarisme, mais qu'il propose également une conception réductrice de ce dernier courant. Il semble aujourd'hui difficile de prendre position pour ou contre "l'utilitarisme" décrit comme un bloc massif, sans prendre en compte l'hétérogénéité des positions que l'on met sous ce nom : on sait que l'utilitarisme "hédoniste" de Bentham n'est pas l'utilitarisme "indirect" de

17 Je n’ai pas de connaissance assez précise des ouvrages plus récents de Frey pour savoir s’il défend encore ces positions ou non.

18 L'auteur réfute les thèses conséquentialistes à partir d'une position kantienne, mais à aucun moment il ne justifie les raisons pour lesquelles il faudrait accorder une supériorité à la morale kantienne. Il ne s'agit pas ici de discuter de l'intérêt de la morale kantienne en elle-même, mais plutôt de constater que sa valorisation par l'auteur relève davantage de la pétition de principe que de la justification rationnelle. 
Mill, ni celui "des préférences" que défend Singer (ainsi que Frey) à la suite de Hare ; l'opposition entre un utilitarisme "de l'acte" et un "de la règle" est aussi régulièrement mentionnée, bien qu'elle soit considérée comme non-pertinente par certains. Or, malgré ses nombreuses références à "l'utilitarisme" (57, rappelons-le), Philippe Larralde ne prend jamais le soin de définir ou de préciser la thèse qu'il met sous ce nom. Un tel souci de précision irait peut-être plus loin que l'objectif de l'auteur, qui n'est pas de discuter l'utilitarisme en lui-même : il semble plutôt que l'objectif, dans la mention de ce courant théorique, soit simplement stratégique, en ce qu'il permettrait, par le jeu d'association qu'il construit (antispécisme-utilitarisme-conséquentialismehétéronomie-morale enfantine), de discréditer par ricochet la position antispéciste. [42]

Néanmoins, si nous souhaitons être précis, alors de deux choses l'une : ou bien il appartient à Philippe Larralde de prouver, premièrement que l'utilitarisme est lié de manière consubstantielle à l'antispécisme, deuxièmement que ce lien serait vrai quels que soient les différents courants d'utilitarisme considérés ; ou bien l'adversaire de Larralde est Singer, et alors le courant qu'il critique est précisément l'utilitarisme des "préférences" ou "intérêts". La première alternative semblant difficile à adopter (en raison de la diversité des fondations métaéthiques de l'antispécisme évoquée dans le point précédent), tentons de confronter l'utilitarisme des préférences aux affirmations de Philippe Larralde, notamment celle, récurrente, de la négation d'une particularité de l'humain :

- « la grande difficulté de l'utilitarisme, sur le plan moral, est, nous l'avons évoqué, le refus de l'idée de la spécificité de l'homme »,

- « dans la mesure où, le seul critère retenu pour appliquer l'impératif moral étant la capacité de jouir et de souffrir, on ne saurait admettre aucune spécificité essentielle de l'homme »,

- «P. Singer les assume avec une grande clarté : ce refus signifie que la valeur de la vie des personnes humaines n'est a priori pas nécessairement plus grande que celle de certains animaux ».

De telles affirmations manqueraient sûrement déjà leur cible dans le cas d'un utilitarisme hédoniste, mais elles le font très clairement si l'on se place dans le cadre de l'utilitarisme des "préférences" ou des "intérêts". En effet, Singer est très explicite sur ce point : il convient de distinguer l'intérêt à ne pas souffrir et l'intérêt à vivre. Le premier type d'intérêt place effectivement sur le même plan toutes les espèces sentientes, puisqu'aucun être capable de ressentir de la douleur ne souhaiterait en ressentir. En revanche, en ce qui concerne l'intérêt à vivre, il est légitime selon Singer de traiter différemment les humains (adultes et sains d'esprit) et les autres espèces animales, notamment en raison de la capacité qu'ont les premiers à se projeter dans l'avenir, à élaborer des projets, qui seraient contrariés par un décès. Singer assume explicitement que l'intérêt à vivre d'un humain est supérieur à celui d'une souris : dire le contraire, c'est soit ne pas l'avoir lu, soit le caricaturer volontairement. Les conséquences concrètes de la position de Singer font qu'il n'est pas abolitionniste : il ne voit pas d'objection au fait de tuer une souris qui n'a pas élaboré de plans pour son avenir, à partir du moment où elle est tuée sans douleur.

Une seconde distinction proposée par Singer permet de concilier l'utilitarisme avec la prise en compte d'une particularité humaine : il s'agit de la différence qu'il propose entre égalité de traitement et égalité de considération (des besoins et intérêts). La seule chose que requiert Singer, c'est une égalité de considération entre les intérêts des différentes espèces, mais puisque celles-ci ont des constitutions et des besoins différents, il est clair qu'une telle égalité ne se manifestera pas concrètement par un traitement similaire d'une espèce à une autre :

« Ce que cette préoccupation ou considération exige précisément que nous fassions peut varier en fonction des caractéristiques de ceux qui sont affectés par nos actes : la préoccupation pour le bien-être des enfants qui grandissent aux États-Unis peut exiger que nous leur apprenions à lire ; la préoccupation 
pour le bien-être des cochons peut ne rien impliquer d'autre que de les laisser en compagnie d'autres cochons dans un endroit où il y a une nourriture suffisante et de l'espace pour courir librement. $»^{19}$

Philippe Larralde aurait dû prendre en compte ces deux distinctions faites par Singer (intérêt à vivre / intérêt à ne pas souffrir ; égalité de considération / égalité de trai-[43]tement), mais la négliger lui permet d'attribuer à Singer des propos qu'il n'aurait jamais tenus, en l'occurrence ceux selon lesquels il serait préférable de tuer un humain diminué plutôt qu'un autre animal en bonne santé. En effet, par la mise en scène d'un dilemme au sein duquel il faudrait choisir entre la vie d'un humain et celle d'un être vivant appartenant à une autre espèce, Philippe Larralde peut dramatiser l'opposition et caricaturer les propos de Singer, et de l'antispécisme en général :

- «Par ailleurs, fidèle au principe utilitariste, P. Singer est partisan de l'euthanasie (à quoi bon maintenir la vie si la somme des souffrances l'emporte sur celle des bonheurs?). Si bien que l'idée de sacrifier une vie humaine apparait, dans certains cas de personnes très diminuées, comme facilement acceptable (en particulier plus acceptable que celle de tuer certains animaux).»

- «Par exemple, selon un tel raisonnement, je peux considérer que la vie d'un malade Alzheimer vaut moins que celle d'un porc adulte en bonne santé. Il serait donc plus rationnel de tuer le malade plutôt que le porc. »

Dans ces situations, Philippe Larralde franchit lui-même le Rubicon qu'il reproche aux utilitaristes de dépasser. En effet une telle comparaison implique l'idée qu'il y aurait comme un choix cornélien à faire entre sauver un mammifère d'élevage et un vieillard ayant perdu conscience. Une telle comparaison ne mériterait un examen moral que dans un cas très précis : imaginons par exemple que nourrir un tel vieillard inconscient de viande fraîche soit la seule manière de le maintenir en vie ; dans ce cas, alors il serait effectivement justifié de mettre en balance la vie de la personne mourante et celle des êtres vivants que l'on utiliserait pour le nourrir. Un tel cas de figure n'obtiendrait sûrement pas de réponse unanime dans la kyrielle des courants de l'antispécisme, néanmoins, puisqu'elle n'est pas à même de se produire, la comparaison par l'auteur entre le vieillard mourant et le mammifère d'élevage en bonne santé ne vise qu'un but rhétorique de dramatisation par la mise en rapport établie entre deux éléments que l'on aurait du mal à comparer (mais qu'il n'est pas nécessaire de comparer dans la vie courante). L'antispécisme se contente de dire qu'il ne faut pas tuer des animaux sentients pour notre simple plaisir gustatif, il ne dit pas qu'il faut tuer des humains malades d'Alzheimer. Et si l'utilitarisme déplace la frontière qui englobe les êtres dont nous devons moralement prendre la douleur en considération, ce n'est pas pour la restreindre aux seuls humains dotés de capacités mentales, mais bien plutôt pour l'élargir à tous les êtres capables de ressentir de la douleur. La bonne foi et le rapport avec la question antispéciste semblent ainsi avoir totalement disparu dans certaines envolées lyriques où l'auteur semble décrire les antispécistes comme des assassins d'humains :

« Par ailleurs la question morale est celle-ci : suis-je bien certain que, si j’étais atteint par cette maladie, j'accepterais qu'autrui puisse juger "objectivement" de la valeur de ma vie ? qu'il puisse éventuellement décider de me tuer parce qu'il a jugé que ma vie était devenue sans valeur? Et cet "autrui", est-il certain qu'il saurait voir les imperceptibles mais parfois déchirants moments d'humanité arrachés à la maladie ?»

Cette présentation complètement faussée de la thèse singerienne culmine dans la reformulation que donne Philippe Larralde de l'argument le plus connu de Singer, celui des cas-limites (et qui est devenu un incontournable dans les discussions sur l'antispécisme) :

« Examinons d'abord les conséquences pratiques du refus utilitariste d'envisager une telle spécificité. P.

Singer les assume avec une grande clarté : ce refus signifie que la valeur de la vie des personnes humaines n'est a priori pas nécessairement plus grande que celle de certains animaux. P. Singer multiplie ici complaisamment les exemples ${ }^{20}$. Si, pour mesurer la valeur d'une vie, l'on retient le critère de la conscience de soi ou celui de la capacité à [44] nouer des liens, il faut affirmer, selon cet auteur, que la vie

19 P. Singer, La libération animale, pp. 71-72. 
d'un nourrisson débile ou d'un vieillard sénile vaut objectivement moins que celle d'un cochon en bonne santé. »

Rappelons l'argument de Singer, qui est un simple raisonnement par l'absurde: son objectif principal est de défendre la thèse suivante : "pour savoir s'il est légitime ou non d'infliger de la douleur à un être vivant, le seul critère à prendre en compte est sa capacité à ressentir une telle douleur", par opposition à celle-ci : "pour savoir s'il est légitime ou non d'infliger de la douleur à un être vivant, le seul critère à prendre en compte est l'intelligence de l'être vivant en question". Ainsi, s'adressant à des antagonistes qui défendraient que ce sont les capacités mentales qui justifient l'exigence de ne pas infliger de la douleur, il raisonne ainsi :

- si jamais l'absence de capacité intellectuelles comparables à celles des humains était véritablement ce qui justifie d'élever, d'enfermer, et de tuer d'autres espèces pour les manger, alors, selon cette maxime, comment faudrait-il traiter les humains qui ne disposent pas non plus de telles capacités intellectuelles (enfants en bas âge, déficients mentaux, personnes âgées séniles, etc.) ?

- en cohérence avec votre principe précédent, l'association entre capacités intellectuelles et traitement moral devrait autoriser à élever, enfermer, et manger également ces individus (et les utiliser pour la recherche), car ils ne disposent pas non plus des capacités intellectuelles que vous tenez pour nécessaires pour justifier un traitement moral respectueux.

- si jamais vous êtes en désaccord avec cette conséquence (ce qui est fort vraisemblable), alors c'est que vous devez réviser votre prémisse initiale selon laquelle ce sont les capacités intellectuelles d'un individu qui déterminent les conditions moralement exigibles quant à la manière de le traiter ${ }^{21}$.

Ainsi, dans la formulation qu'en donne Philippe Larralde, il semble qu'il ait pris au premier degré ce qui chez Singer consiste la conséquence volontairement absurde d'un raisonnement qui vise à faire rejeter la prémisse sur laquelle il repose. Une erreur aussi manifeste d'interprétation discrédite non seulement le contre-argument de l'auteur, mais jette un sérieux doute sur sa connaissance ou sa rigueur de lecture par rapport aux courants qu'il entend critiquer, ainsi que sa capacité même à identifier un raisonnement par l'absurde. Enfin, là où la question du traitement des cas limites n'a rien à voir avec l'antispécisme, c'est qu'un auteur utilitariste et spéciste comme Raymond Frey accepte l'expérimentation sur les cas-limites humains (position qui, bien entendu, n'a pas manqué de faire réagir).

\section{L'euthanasie et la barque : des exemples auto-réfutants ?}

Dans son entreprise d'opposition à un antispécisme qu'il perçoit comme nécessairement associé à l'utilitarisme, Philippe Larralde présente deux arguments qu'il considère comme fatals contre l'utilitarisme : celui de l'euthanasie et celui de la barque. Or, comme nous le verrons, non seulement il n'est pas évident que de tels arguments s'opposent vraiment à l'utilitarisme, mais de plus, si l'on en revient au sujet initial qui [45] est non l'utilitarisme mais bien l'antispécisme, alors il se pourrait bien que les arguments en question soient en fait favorables à la position que l'auteur entend critiquer.

20 Larralde déclare que Singer « assume avec une grande clarté » et « multiplie [...] complaisamment les exemples », il aurait donc vraisemblablement pu être en mesure de fournir rien qu’une seule citation où Singer présenterait explicitement cette position.

21 On pourrait ajouter une dernière conséquence qui permet de définir le spécisme : et si vous dites "bien que les humains déficients mentaux ne disposent pas plus de ces capacités intellectuelles que d'autres espèces, il faudrait néanmoins les traiter différemment, non parce qu'ils sont ou pourraient être plus intelligents, mais au simple motif qu'ils sont humains", alors, en érigeant la frontière interespèce comme critère arbitraire d'un traitement différencié, vous manifestez une position que l'on nommera spéciste. 
Afin de montrer les insuffisances de la position utilitariste, l'auteur prend l'exemple de l'euthanasie : il entend ainsi renvoyer dos à dos deux positions qu'il juge «extrêmes »: d'un côté un positionnement « religieux » (quelle religion?), qui condamne absolument toute euthanasie car toute vie serait sacrée, et de l'autre, l'utilitarisme qui l'autoriserait de manière débridée. La position dite "humaniste" qu'il défend se présente comme un compromis entre ces deux " extrêmes » et se formule selon le principe suivant : on ne serait légitime à évaluer moralement la vie d'un individu que lorsque celui-ci est privé de sa conscience. Or, si l'on renvoie cette discussion parenthétique à l'objet initial de l'argumentation de l'auteur, on est tenté de se demander : en quoi cette position finale est-elle opposée à la thèse antispéciste ? À supposer que la notion de "conscience" ait un sens qu'il soit possible de définir, ne dit-on pas justement que c'est ce dont les espèces non-humaines seraient démunies ? Et dans ce cas, puisque la position de l'auteur consiste à dire qu'il est légitime d'évaluer d'un point de vue utilitariste la valeur de la vie d'un individu privé de sa conscience ${ }^{22}$, n'est-il pas précisément en train de dire qu'il est légitime d'évaluer la valeur de ces être sans conscience que seraient les animaux non-humains ? Bref, il semble bien qu'en voulant contredire la position antispéciste, l'auteur la renforce, sans même s'en rendre compte, par son argument de l'euthanasie. Par ailleurs, c'est bien la focalisation sur l'utilitarisme qui conduit l'auteur à penser que la question de l'euthanasie d'humains aurait une quelconque conséquence sur celle de l'antispécisme. En effet, si l'on s'appuyait sur la notion de "sujet d'une vie", mise en avant par Tom Regan (rappelons que cet auteur est aussi important que Singer dans la fondation théorique de l'antispécisme contemporain) comme fondement à sa théorisation de l'antispécisme, alors il serait tout à fait possible de défendre une position anti-euthanasie compatible avec l'antispécisme.

L'exemple de la barque n'est pas plus convaincant. L'auteur déclare que, dans une situation où l'on aurait le choix entre sacrifier une personne pour alléger une barque et en sauver 100, ou prendre le risque de faire couler la barque, alors l'utilitarisme serait capable de dire qui il faut sacrifier. Pour commencer, rappelons que le dilemme de la barque n'est pas spécifiquement une attaque antiutilitariste : il s'agit d'une expérience de pensée en morale que l'on connaît au moins depuis la planche de Carnéade, et qui a connu récemment des formulations plus contemporaines (mobilisant plutôt un train ou un tramway sur des rails) depuis Philippa Foot. En mettant en scène une situation non-quotidienne (espérons-le !) où nous serions sommés de choisir entre la vie d'une personne et celle de plusieurs autres, elle oblige à clarifier les fondements éthiques au nom desquels les actions sont entreprises.

Premièrement, l'auteur est toujours aussi évasif qu'à son habitude lorsqu'il déclare simplement que « D'un point de vue utilitariste conséquent, il est rationnel donc objectivement moral de sacrifier une personne », sans pouvoir proposer de référence, ou préciser [46] le courant utilitariste dont il parle. Deuxièmement, est-il vraiment impossible de concevoir qu'un point de vue dit "humaniste" défende également le fait de sauver 99 « fin[s] en soi » plutôt que de n'en sauver qu'une ? Dans ce cas précis, il semble que l'auteur n'a pas distingué entre deux dimensions de la question : d'une part, la question de savoir s'il faut sacrifier 1 personne pour que les 99 autres puissent vivre ; d'autre part, celle de savoir sur quel critère sera opéré le choix de la personne à sacrifier (une quantification utilitariste de la valeur de la vie de chacun, un tirage au sort, une décision arbitraire assumée de type "la dernière personne à avoir embarqué ne sera pas acceptée", etc.).

22 «Mais, on le sait, il existe un cas limite : que faire lorsque la personne n’est plus consciente ? C'est en ce point précis qu'intervient le principe d'utilité : dans la mesure où cette personne a perdu de fait sa capacité de juger de la valeur de sa propre vie, l'entourage se trouve condamné à juger à sa place. Il devient alors incontournable d'effectuer une opération qui est impossible (et moralement inadmissible) dans l'absolu : évaluer objectivement la valeur de la vie d'autrui. C'est parce que le principe absolu du respect du jugement d'autrui est inapplicable, que l'on est contraint à « bricoler » un jugement qui soit le moins arbitraire possible concernant la valeur d'une vie humaine. C'est le seul cas où le calcul des plaisirs et des peines, aussi absurde soit-il en général, peut avoir un sens. Dans cette (unique) circonstance, le principe d'utilité vient limiter celui du caractère sacré du respect du jugement d'autrui sur la valeur de sa propre vie et interdire l'acharnement thérapeutique. » 
Et surtout, encore une fois, bien que ce raisonnement vise à s'opposer à une posture utilitariste, il ne constitue finalement pas une objection solide contre l'antispécisme lui-même : dans le cas de la consommation de viande, l'antispécisme déclare qu'il est illégitime de sacrifier la vie d'un être vivant, non pas pour la survie de 99 autres, mais pour le simple plaisir gustatif (ou la continuité envers des habitudes de consommation acquises) de quelques autres ${ }^{23}$. Et précisément, la posture de l'antispécisme consiste à dire que, même si l'on pouvait quantifier la quantité de plaisir que procure le fait de manger de la viande, celle-ci ne pourrait jamais être considérée comme comparable à l'intérêt d'un être vivant à ne pas subir les conditions de l'élevage. Par conséquent, il semble ici aussi cet exemple conforte la thèse antispéciste davantage qu'il ne la conteste.

\section{La reductio ad Hitlerum}

Le dernier point problématique dans l'argumentation de Philippe Larralde réside dans son association de l'antispécisme au nazisme, ceci pour trois raisons: premièrement, c'est historiquement faux ; deuxièmement, car la mention d'un point commun avec le nazisme ne peut nourrir un discrédit que sous des conditions logiquement spécifiques qui ne sont pas réunies ici ; troisièmement, car ce procédé manque d'honnêteté et révèle généralement l'absence d'autres arguments plus sérieux. Commençons par relever les passages au sein desquels l'auteur mobilise une telle comparaison:

- «Ce n'est pas un hasard si, comme le note L. Ferry, P. Singer a été interdit de parole en Allemagne : ses idées y ont été interprétées comme un appel au meurtre des aliénés (terrible souvenir, bien sûr, des crimes du nazisme).»

- «Mais, si l'on veut prendre la mesure de ce problème, le plus édifiant est sans doute d'avoir à l'esprit qui sont, sur un plan politique, les précurseurs de la défense des animaux : ce sont les nazis qui ont promulgué le 24 novembre 1933 une loi novatrice sur la protection des animaux (Tierschutzgesetz). Hitler, inspirateur de cette loi, a pu déclarer : "Dans le nouveau Reich il ne devra plus y avoir de place pour la cruauté envers les bêtes." [cit. L. Ferry] »

- «Hitler est ainsi incontestablement un pionnier de la cause animale. Cela ne signifie pas, bien sûr, que l'amour des animaux entraîne nécessairement la haine des hommes, mais que ce n'est nullement incompatible et que ce danger découle directement d'une perspective philosophique qui, étendant la préoccupation morale aux animaux, repose sur la négation de la spécificité de l'homme. Cette négation utilitariste est donc bien le fondement de la régression antihumaniste. »

- «Simplement un exemple qui fait tout de même froid dans le dos lorsque l'on songe aux déportations des juifs par les nazis. »

- «Habituellement tous les mouvements intellectuels ont a cœur de rendre hommage à leurs précurseurs. Or ici, non seulement personne, chez les défenseurs des animaux, ne rend justice à l'œuvre très consistante de Hitler, mais il y a manifestement une volonté de la dissimuler. C'est pourquoi il faut l'affirmer très clairement : la défense utilitariste [47] des animaux est le fondement d'une profonde et très dangereuse régression antihumaniste. On est donc infiniment loin de la niaiserie selon laquelle étendre la préoccupation morale aux animaux ferait accéder l'humanité à un stade éthique supérieur : cela peut aussi (dès que l'on refuse l'idée d'une spécificité de l'homme) ouvrir la voie à la pire barbarie. »

La première objection à l'encontre de cette association entre antispécisme et nazisme est qu'elle est factuellement fausse. Elle est reprise à l'ouvrage de Luc Ferry intitulé Le nouvel ordre écologique (Grasset, 1992), ainsi qu'à son recueil de textes Des animaux et des hommes, co-édité avec Claudine Germé (Livre de Poche, 1994); or des études historiques comme celle d'Élisabeth Hardouin-Fugier ${ }^{24}$ ont pu montrer de manière rigoureuse et convaincante que ces allégations étaient fausses d'un point de vue historique. Si la propagande nazie a bien pu vanter le régime de s'être

23 Le rapport quantitatif va d'ailleurs plutôt dans l'autre sens, vu le nombre d'animaux qu'il est nécessaire de tuer pour nourrir un humain non-végétarien sur toute une vie. 
opposé à la maltraitance des animaux non-humains (parmi la totalité des autres allégations sur lesquelles il est possible de faire de la propagande), le dispositif légal n'a fondamentalement rien modifié au traitement des animaux non-humains sous le $\mathrm{III}^{\mathrm{e}}$ Reich. Les affirmations de Luc Ferry à ce sujet ne peuvent valoir qu'à partir d'une prise en compte au premier degré de ces éléments de propagande ; sa présentation historique de la question a par ailleurs présenté comme loi un texte qui n'en était que le commentaire, Ferry ajoutant la signature d'Hitler dans la reproduction qu'il donne du document; la totalité de ses remarques vont dans le sens de présenter Hitler comme personnellement impliqué dans cette cause, alors que les documents étudiés par Élisabeth HardouinFugier montrent que ce n'est pas le cas.

$\mathrm{Au}$ contraire, comme le souligne Jean-Baptiste Jeangène Vilmer, différents auteurs ont remarqué un lien entre le traitement actuel des animaux non-humains et l'idéologie nazie au moins sur trois points $^{25}$ : le premier réside dans la dénégation d'humanité d'une certaine partie de la population (les Juifs, homosexuels, gens du voyage, personnes racisées, etc.) qui autorise à les traiter "comme des animaux" ${ }^{16}$, le second réside dans l'influence qu'aurait eue la pratique des abattoirs aux États-Unis sur les solutions d'extermination dans les camps, par l'intermédiaire du fordisme, le troisième tient dans l'idéologie eugéniste qui est à l'œuvre aussi bien dans le programme du nazisme que dans la pratique des croisements et de la sélection des espèces au sein de l'élevage.

Par ailleurs, les citations en question regorgent de confusions ou d'erreurs factuelles sur bien d'autres points. Plusieurs travaux historiques démentent l'idée qu'Hitler ait été végétarien ${ }^{27}$. À la lecture de certaines phrases de Philippe Larralde ${ }^{28}$, on se demande s'il [48] faudrait en venir à considérer Hitler comme un utilitariste. Et mentionner qu'Hitler aurait été un " précurseur[ ] de la cause animale renvoie à négliger la totalité des réflexions éthiques qui ont porté sur les animaux non-humains avant les années 1930. D'un point de vue législatif, Élisabeth Hardouin-Fugier remarque qu'une première législation anglaise avait déjà été adoptée dès 1876, et deux actes prussiens en 1885 et 1930, parmi d'autres. D'un point de vue philosophique, rappelons que, si l'on s'appuie sur Empédocle et sur Ovide, alors il est permis de croire qu'il existe une justification philosophique au végétarisme depuis l'école pythagoricienne, et il serait bien trop long ici de citer tou·te·s les philosophes qui ont traité de l'animalité avant Hitler $^{29}$.

La seconde objection que l'on peut formuler à l'encontre de cette association réside non sur sa factualité mais sur ses fonctions rhétoriques dans l'argumentation de Philippe Larralde. En voulant

24 É. Hardoin-Fugier, « La protection législative de l'animal sous le nazisme. Un recyclage français de la propagande nazie (autour des ouvrages de Luc Ferry) », in Luc Ferry ou le rétablissement de l'ordre. L'humanisme est-il antiégalitaire ?, éd. Tahin Party, pp. 127-151. Le texte est disponible ici : http://tahin-party.org/textes/ferry127-151.pdf. Voir aussi, R. Kalechofsky, «Les nazis, les animaux, et l'expérimentation animale », in Cahiers antispécistes $\mathrm{n}^{\circ} 18$ février 2000, http://www.cahiers-antispecistes.org/les-nazis-les-animaux-et-lexperimentation-animale/

25 J.-B. Jeangène Vilmer, Éthique Animale, PUF, 2008, pp. 40-43.

26 L'ouvrage le plus connu à ce sujet, et qui a suscité de nombreux débats, est : Ch. Patterson, Eternal Treblinka, Lantern Books, 2002.

27 On peut reprendre ici la référence à Animals' Agenda, mai 1992, p. 31, donnée par David Olivier dans son article Luc Ferry ou le rétablissement de l'ordre (Cahiers Antispécistes, n5, décembre 1992, note 16. http://www.cahiersantispecistes.org/luc-ferryou-le-retablissement-de-lordre/) : " L'historien végétarien Rynn Berry a réfuté le mythe selon lequel Adolf Hitler aurait été végétarien, en documentant le goût de ce dernier pour les saucisses bavaroises, le jambon, le foie et le gibier, ainsi que l'interdiction qu'il édicta à l'encontre des organisations végétariennes dans tous les territoires contrôlés par les nazis. La chef cuisinière Dione Lucas, qui cuisinait souvent pour Hitler, mentionna son goût pour le pigeon farci en page 83 de son ouvrage The Gourmet Cooking School Cookbook. ».

28 « Hitler est ainsi incontestablement un pionnier de la cause animale. Cela ne signifie pas, bien sûr, que l’amour des animaux entraîne nécessairement la haine des hommes, mais que ce n'est nullement incompatible et que ce danger découle directement d’une perspective philosophique qui, étendant la préoccupation morale aux animaux, repose sur la négation de la spécificité de l’homme. Cette négation utilitariste est donc bien le fondement de la régression antihumaniste. »

29 On pourra se référer à J.-B. Jeangène Vilmer, op. cit., pp. 21-40, et É. de Fontenay, 1998, Le silence des bêtes, Fayard. 
associer les antispécistes et végétariens à Adolf Hitler, l'auteur entend produire un mouvement de rejet par contagion : si l'on est contre Hitler (qui ne l'est pas ?), et si celui-ci avait la propriété X, alors il faut aussi être contre la propriété X. Une telle association ne peut valoir rigoureusement que si l'on peut montrer que la propriété $X$ est intrinsèquement liée à l'essence du nazisme. Ainsi, si la propriété en question est "être antisémite", il est probable que le raisonnement soit valide. En revanche, si la propriété considérée chez Hitler est "aimer la musique de R. Wagner" ou "porter une moustache", il est probable que l'association avec Hitler ne sera d'aucune portée pour l'appréciation ou le rejet de la propriété en question. Ainsi, l'association de Philippe Larralde vaut surtout pour le présupposé sur lequel elle repose, puisque pour qu'elle soit valable, il faut qu'il existe un lien intrinsèque et essentiel entre le végétarisme (ou l'antispécisme) et le nazisme. Mais précisément, la présentation qu'il en donne tend à tenir pour acquis ce qu'il s'agissait de prouver (à savoir, l'existence d'un tel lien intrinsèque). Pour comprendre la difficulté d'un tel raisonnement, on peut prendre un autre exemple : la marque de voiture Volkswagen est née sous le III ${ }^{\mathrm{e}}$ Reich, et répond à la volonté qu'avait Hitler de doter tout·e citoyen·ne allemand·e d'une voiture : est-ce à dire qu'aujourd'hui, toute personne conduisant une voiture de cette marque devrait se voir suspectée de collusion avec le nazisme ${ }^{30}$ ?

La troisième objection s'interroge sur les intentions que manifeste le recours au procédé de comparaison avec le nazisme, et sur la possibilité qu'a l'auteur de faire appel à des arguments plus consistants. Une telle comparaison renvoie à une forme typique d'argument qui a été nommé ironiquement la "reductio ad Hitlerum" (on parle plutôt de "point Godwin" dans le cas de échanges sur internet), et dont voici une définition provenant de Wikipedia ${ }^{31}$ :

« La reductio ad Hitlerum consisterait par exemple, à rejeter les campagnes anti-tabac du fait que Hitler les soutenait en son temps, ou à critiquer le végétarisme parce qu'Hitler était végétarien.

Cette tactique rhétorique a pour objet d'exclure l'adversaire du champ polémique tout en évitant le débat de fond. Avec d'autres techniques rhétoriques, comme l'argumentation ad hominem, la reductio ad Hitlerum apparaît généralement à bout de discussion, lorsque les adversaires ont épuisé toutes les preuves et tous les arguments rationnels pour se convaincre mutuellement.

L'expression est apparue pour la première fois en 1951 dans un article du philosophe Leo Strauss pour la revue Measure : a critical journal et a été reprise et popularisée en 1953 [49] dans son livre Natural Right and History. Elle a été réutilisée plus tard par le philosophe spécialiste de la Shoah George

Steiner. »

Bref, on remarque qu'une telle référence grève davantage la crédibilité de la partie qui en fait usage que de celle qui est visée.

\section{Conclusion}

L'objectif de cet article n'est pas de proposer une liste exhaustive des difficultés que présente celui de Philippe Larralde. Je n'ai rien de particulier contre cet auteur, que je ne connais pas personnellement. Par ailleurs, j'espère que l'on aura bien compris que cette critique ne repose pas sur un attachement particulier à l'utilitarisme ou à un militantisme antispéciste dogmatique, mais qu'elle vise plutôt à analyser les modalités de raisonnement mobilisées par l'auteur. Une telle mise au point me semblait nécessaire dans la mesure où les confusions de son article sur lesquelles je me suis arrêté sont véhiculées dans de nombreux autres discours concernant l'antispécisme : à ce titre, c'est moins la personne que je vise ici, que des propos relativement répandus, que l'on peut trouver ailleurs. La source principale de ces erreurs est bien souvent l'ouvrage de Luc Ferry, Le nouvel

30 Voir aussi à ce sujet : D. Olivier, « Luc Ferry ou le rétablissement de l'ordre », Cahiers Antispécistes, n5, décembre 1992, http://www.cahiers-antispecistes.org/luc-ferryou-le-retablissement-de-lordre/. L’argument précis se situe pp. 42-52 dans l'édition de cet article chez Tahin Party : http://tahin-party.org/textes/ferry37-79.pdf

31 https://fr.wikipedia.org/wiki/Reductio ad Hitlerum 
ordre écologique, déjà cité. La première erreur que commet cet ouvrage fut d'avoir associé la revendication antispéciste à un mouvement écologiste ${ }^{32}$. La plupart des erreurs que nous avons identifiées dans l'article de Larralde ne sont ainsi pas de son fait, mais se trouvent déjà dans l'ouvrage de Ferry. Reprenons rapidement la liste de celles-ci :

- L'antispécisme ne porte pas sur la question de la différence ou de l'identification entre espèces humaine et non-humaines, mais seulement sur l'égale considération des différentes espèces à ne pas ressentir de la douleur.

- L'antispécisme ne peut pas être réduit à l'utilitarisme car d'une part il existe un grand nombre de courants théorisant l'antispécisme sur une base autre qu'utilitariste (par exemple Tom Regan), d'autre part il est possible de tirer des conséquences spécistes de l'utilitarisme (comme le fait Raymond Frey).

- En vertu de la distinction entre "agent moral" et "patient moral", on ne peut pas dire qu'attribuer des droits à un individu revient à lui imputer des devoir, puisqu'il existe un certain nombre d'individus ne pouvant être que patients moraux sans être agents moraux.

- Il n'est pas possible de confondre dans une même formulation une défense utilitariste de l'antispécisme et une défense qui invoquerait un "droit des animaux", car cela renvoie à deux courants concurrents (incarnés par Singer d'une part, Regan de l'autre).

- Il n'est pas possible de parler de "l'utilitarisme" en général car celui-ci renvoie à des courants hétérogènes et divergents. En particulier, pour le cas qui nous concerne, Singer mobilise un "utilitarisme des préférences" qui ne rend pas comparables les expériences humaines et nonhumaines du point de vue de l'intérêt à vivre (car les humains [50] ont une capacité à se projeter dans l'avenir), mais seulement de celui de l'intérêt à ne pas ressentir de douleur.

- L'argument des cas-limites formulé par Singer est un argument par l'absurde : Singer ne dit pas qu'il faut enfermer dans des cages pour les manger des humains ne disposant pas de notre rationalité (bébés, déficients mentaux, personnes séniles, etc.), mais plutôt que si l'on refuse une telle pratique, alors il faut admettre que ce n'est pas la rationalité d'une être vivant qui fonde sa respectabilité morale.

- L'association entre l'antispécisme et le nazisme est factuellement fausse, logiquement peu rigoureuse, et témoigne surtout d'un manque d'arguments véritables.

D'une certaine manière, on pourrait dire que la présence de ces confusions déjà dans l'ouvrage de Ferry disculpe Philippe Larralde de leur invention. Le seul reproche qu'il resterait à faire à ce dernier est simplement de s'être laissé piéger par les approximations du Nouvel ordre écologique et de n'avoir pas cherché à compléter sa réflexion par des lectures complémentaires ${ }^{33}$. Il aurait en effet suffi à l'auteur d'ouvrir le ch. 1 de La libération animale, de Peter Singer, pour dissiper beaucoup de ces confusions. Pour revenir sur l'agacement suscité par l'usage que Philippe Larralde a fait de mon propre article, j'espère qu'il n'est pas causé par un quelconque ego mal placé, et je peux maintenant en préciser deux raisons : la première est qu'il a permis à l'auteur de manifester à peu de frais une

$32 \mathrm{Si}$, aujourd'hui, ces deux revendications se rapprochent, et il n'est pas rare de voir des personnes refusant de manger de la viande à la fois pour des raisons environnementales (la production de viande, en particulier de bœuf, nécessite des quantités phénoménales d'énergie et d'eau, elle est une source importante de rejets de méthane, un gaz dont l'effet de serre est plus fort que le $\mathrm{CO}_{2}$, ; enfin, à quantité égale de nourriture, la culture de végétaux (y compris fournissant des protéines) nécessite une superficie largement moindre, et contribue donc moins à la déforestation) et éthiques, il faut re-situer le débat dans son contexte historique : en 1992, quand paraît l'ouvrage de Ferry, nombre d'antispécistes ne sont pas écologistes, et considèrent même ces derniers comme des adversaires théoriques (cf. par exemple Pourquoi je ne suis pas écologiste, rédigé par David Olivier en 1988 et publié dans les Cahiers Antispécistes en 1993, http://www.cahiers-antispecistes.org/pourquoi-je-ne-suis-pas-ecologiste/).

33 On peut néanmoins s'interroger sur la démarche méthodologique visant à critiquer un courant de pensée en ne se fondant que sur les écrits de ses détracteurs : considérerait-on comme sérieuse une critique du marxisme qui cite pour seule référence des ouvrages de Hayek ? La situation est pourtant comparable ici, l'ouvrage de Ferry étant ouvertement hostile à l'antispécisme, il a d'ailleurs suscité un certain nombre de réactions de la part de ce courant. Plusieurs de celles-ci ont été regroupées dans un ouvrage publié chez Tahin Party et disponible sur internet : http://tahin-party.org/ferry.html 
connaissance de l'antispécisme, en y puisant quelques citations par une lecture hâtive et décontextualisée, qui l'ont, semble-t-il, dispensé de lire les originaux (comme je l'ai déjà mentionné, la totalité des citations antispécistes de son article étaient présentes dans le mien), la deuxième raison est que mon article mentionnait déjà quelques distinctions classiques de l'éthique animale, qui auraient permis à l'auteur d'éviter quelques contresens : l'auteur semble donc s'être autorisé à y puiser simplement 3 citations en négligeant la plupart des définitions et distinctions qui fondent le cadre définitionnel du courant qu'il entend critiquer ${ }^{34}$.

À toute personne qui souhaiterait se positionner dans les débats complexes et passionnants au sujet de l'éthique animale sans retomber dans les travers que j'identifie ici, je pourrai recommander quelques pistes bibliographiques, et introductions au domaine, qui constitueraient un bon point de départ :

- H.-S. Affeissa \& J.-B. Jeangène Vilmer, (éd.), 2010, Philosophie Animale, Vrin, collection "textes clés". [50]

- J.-B. Jeangène Vilmer, «Les principaux courants en éthique animale », in. La question animale, entre science, littérature et philosophie, PUR, 2011, pp. 79-92 (disponible aussi ici : http://www.jbjv.com/Les-principaux-courants-en-ethique.html)

- J.-B. Jeangène Vilmer, Éthique Animale, PUF, 2008

- É. de Fontenay, 1998, Le silence des bêtes, Fayard.

- La revue Cahiers antispécistes, dont tous les numéros sont lisibles en-ligne. Cette revue a traduit, à la fin du $\mathrm{XX}^{\mathrm{e}}$, des textes classiques et incontournables de la discussion antispéciste, et elle fait une large part aux désaccords internes entre les courants antispécistes. http://www.cahiers-antispecistes.org/

- P. Singer, 1975, La libération animale, trad. éd. Payot 2012.

- T. Regan, 1983, The Case for Animal Rights, University of California Press, trad. éd. Hermann, 2013.

34 En particulier : la différence entre la question logique de la différence entre espèces et celle, politique, de leur traitement (pp. 259-261), la distinction, chez Singer, entre intérêt à vivre et intérêt à ne pas ressentir de la douleur (pp. 253-254) ; celle entre "égalité de traitement" et "égalité de considération" (pp. 250-254) celle entre "agent moral" et "patient moral" (pp. 271-275) ; une présentation claire du fait que l’argument singerien des "cas-limites" est un argument par l'absurde (pp. 265-268), des précisions concernant le fait que l'antispécisme ne se réduit pas à l'utilitarisme (p. 256), etc. Au sujet de la distinction entre "agent moral" et "patient moral", il est intéressant de remarquer qu'elle est présente dans la section IV de l'article de Philippe Larralde, ce qui ne l'empêche pas de dire dans la section II que l'attribution de "droits" aux animaux impliquerait nécessairement de leur imputer des "devoirs" : cette distinction a donc été saupoudrée en fin d'article sans que l'auteur ne prenne la peine de reconsidérer son propos initial en exploitant ses conséquences dans sa section II. Il en va de même pour les citations qu'il produit de Singer à partir de mon article dans sa section IV, qui montrent que l'auteur sait que Singer ne dit pas explicitement préférer la vie d'une souris à celle d'un humain, en revanche il n'en tire pas pour conséquence de nuancer ses propos réducteurs sur l'utilitarisme dans la section III. 